\title{
Communication
}

\section{Stereochemistry of insect kinin tetrazole analogues and their diuretic activity in crickets ${ }^{\star \star}$}

\author{
Ronald J. Nachman ${ }^{1 凶}$, Geoffrey M. Coast ${ }^{2}$, Krzysztof Kaczmarek ${ }^{1,3}$, \\ Howard J. Williams ${ }^{4}$ and Janusz Zabrocki ${ }^{1,3}$
}

${ }^{1}$ Areawide Pest Management Research Unit, Southern Plains Agricultural Research Center, ARS, U.S. Department of Agriculture, College Station, TX, U.S.A.; ${ }^{2}$ School of Biological and Chemical Sciences, Birkbeck College, London, U.K.; ${ }^{3}$ Institute of Organic Chemistry, Technical University of Lodz, Łódż, Poland; ${ }^{4}$ Department of Chemistry, Texas A\&M University, College Station, TX, U.S.A.

Received: 31 October, 2003; revised: 23 January, 2004; accepted: 03 February, 2004

Key words: tetrazole, cis-peptide bond, $\beta$-turn mimetic, constrained insect kinin analogue, diuresis

\begin{abstract}
Insect kinin analogues of the sequence Phe-Phe- $\psi\left[\mathrm{CN}_{4}\right]-\mathrm{Ala}-\mathrm{Trp}-\mathrm{Gly}-\mathrm{NH}_{2}$ containing $\left(\mathrm{L}-\mathrm{Phe}^{2}, \mathrm{~L}-\mathrm{Ala}^{3}\right)$ and (L-Phe $\left.{ }^{2}, \mathrm{D}-\mathrm{Ala}^{3}\right)$ stereochemical variants of the tetrazole moiety, a mimic of the type VI $\beta$-turn, demonstrate significant agonist and partial antagonist activity, respectively, in a cricket diuretic bioassay. A comparison of the solution conformations of these two stereochemical variants indicates a structural basis for their divergent bioactivities. The (D-Phe ${ }^{2}, \mathrm{D}-\mathrm{Ala}^{3}$ ) stereochemical variant was synthesized and found to demonstrate significant agonist activity. The results further define stereochemical requirements for the diuretic activity of insect kinins in crickets and provide valuable information for the design of biostable analogues capable of disrupting digestive and diuretic processes in pest insects.
\end{abstract}

\footnotetext{
${ }^{\star}$ Presented at the 17th Polish Peptide Symposium, August 31st-September 4th, 2003, Łódź, Poland.

This work was supported by the U.S. Department of Agriculture (R.J.N., K.K., G.M.C., J.Z.), a Collaborative Research Grant (LST.CLG.979226) from the North Atlantic Treaty Organization (NATO) (R.J.N., K.K., G.M.C., J.Z.), grant \#000517-0103-2001 from the Texas Advanced Technology/Advanced Research Program (R.J.N., H.J.W.), and by the State Committee for Scientific Research (KBN, Poland) (K.K., J.Z.).

${ }^{\otimes}$ To whom correspondence should be addressed: Ronald J. Nachman, Areawide Pest Management Unit, Southern Plains Agricultural Research Center, U.S. Department of Agriculture, 2881 F\&B Road, College Station, TX 77845 U.S.A.; phone: (01 979) 260 9315; fax: (01 979) 260 9377; e-mail: nachman@tamu.edu
}

Abbreviations: MALDI-TOF-MS, matrix-assisted laser desorption ionization-time of flight mass spectrometry. 
Insect kinins share a highly conserved C-terminal pentapeptide sequence Phe-Xaa-XbbTrp-Gly- $\mathrm{NH}_{2}$, where Xaa can be Tyr, His, Ser or Asn, and Xbb can be Ala but is usually Ser or Pro (Holman et al., 1999). They have been isolated from a number of insects, including species of Dictyoptera, Lepidoptera, and Orthoptera. The first members of this insect neuropeptide family were isolated on the basis of their ability to stimulate contractions of the isolated cockroach hindgut (Holman et al., 1999), but they are also potent diuretic peptides that stimulate the secretion of primary urine by Malpighian tubules, organs involved in the regulation of salt and water balance. More recently, insect kinins, and/or analogues, have been reported to inhibit weight gain by larvae of the tobacco budworm (Heliothis virescens) and corn earworm (Helicoverpa zea) (Nachman et al., 2002a), both serious agricultural pests.

Structurally, insect kinins require an intact C-terminal pentapeptide sequence for full cockroach myotropic and cricket diuretic activity, which therefore represents the active core (Nachman \& Holman, 1991). An Ala-replacement analog series of the insect kinin active core region confirms the importance of the Phe and Trp sidechains, because these are the only two replacements which lead to complete loss of myotropic and diuretic activity (Nachman et al., 1998). Analysis of the conformations adopted by the head-to-tail, cyclic insect kinin analog cyclo(Ala-Phe-Phe-ProTrp-Gly), in which distance and angle constraints obtained from aqueous NMR spectra were incorporated into molecular dynamics calculations, indicated the presence of two turn types over two distinct sets of residues within the active core pentapeptide. The more rigid of the two conformations featured a cisPro in the third position of a type-VI $\beta$-turn over core residues 1-4, or Phe-Phe-Pro-Trp. The other less rigid turn system involved a transPro and encompassed residues 2-5, or Phe-Pro-Trp-Gly. From unrestrained molecular dynamics calculations, the most favorable
cisPro structure had an intramolecular energy about $7 \mathrm{kcal} /$ mole lower than the most favorable transPro structure, consistent with NMR data that indicated that the cisPro structure was the predominant conformation in solution by a 60:40 ratio (Nachman et al., 1990; Roberts et al., 1997).

Although the available evidence (including structure-activity studies) was most supportive of the 1-4 $\beta$-turn as the active receptor bound conformation (Nachman et al., 1990; Roberts et al., 1997), the $2-5 \beta$-turn could not be dismissed as a candidate. In order to obtain more evidence for one of the two available conformations as the active one, an analogue containing a tetrazole moiety (Phe-Phe$\psi\left[\mathrm{CN}_{4}\right]$-Ala-Trp-Gly-NH${ }_{2}$ ) (Fig. 1), that could

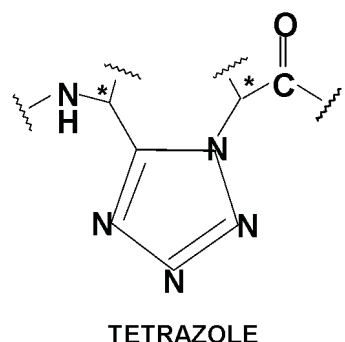

TETRAZOLE

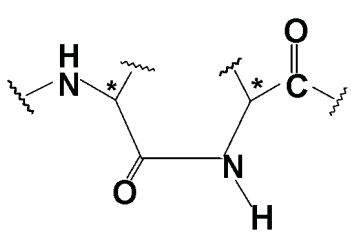

CIS-PEPTIDE BOND
Figure 1. Structure of the tetrazole moiety (left), a mimic of a cis-peptide bond (right) and a type VI $\beta$-turn.

Asterisks delineate chiral centers, which can be either 'LL', 'LD', or 'DD' in the insect kinin analogues included in this study (Table 1).

preferentially form the 1-4 type VI $\beta$-turn (Zabrocki et al., 1988), was synthesized and found to be active in a cricket diuretic assay in the physiological range. A combination of NMR spectroscopic and computer modeling studies indicated that the tetrazole analogue induced a 1-4, type VI turn in aqueous solution, and that formation of the alternative 2-5 turn was energetically unfavorable (Nachman et al., 2002b). Conversely, a stereochemical variant of the tetrazole analogue Phe-Phe- $\psi\left[\mathrm{CN}_{4}\right]$-Ala-Trp-Gly-NH $\mathrm{N}_{2}$ with (L-Phe ${ }^{2}, \mathrm{D}-\mathrm{Ala}^{3}=$ 'LD') stereochemistry proved 
to elicit partial antagonism of the diuretic response of the natural insect kinins in the cricket Malpighian tubule assay (Nachman et al., 2002b). This partial antagonist activity indicated that the ' $\mathrm{LD}$ ' tetrazole analogue could also bind with, but could not fully activate, the insect kinin receptor. These two analogues provided strong evidence for the 1-4, type VI $\beta$-turn as the active conformation for the insect kinins.

Here we report on the aqueous solution conformation of the 'LD' stereochemical variant of the tetrazole analogue Phe-Phe- $\psi\left[\mathrm{CN}_{4}\right]-$ Ala-Trp-Gly- $\mathrm{NH}_{2}$, and a comparison with that of the 'LL' version, in order to identify possible structural explanations for the change in biological activity from an agonist to a partial antagonist. In an effort to further define stereochemical requirements for both binding and activation of the insect kinin receptor, we also report on the synthesis and biological evaluation of a third stereochemical variant, Phe-D-Phe- $\psi\left[\mathrm{CN}_{4}\right]$-D-Ala-Trp-Gly$\mathrm{NH}_{2}$.

\section{MATERIALS AND METHODS}

Chemistry. Synthesis of the desired peptide Phe-D-Phe- $\psi\left[\mathrm{CN}_{4}\right]$-D-Ala-Trp-Gly- $\mathrm{NH}_{2}$ was achieved by fragment condensation on the polymeric support (MBHA type resin) using TBTU \{O-(benzotriazol-1-yl)- $N, N, N^{\prime}, N^{\prime}$-tetramethyluronium tetrafluoroborate $\}$ as a coupling reagent. The tetrazole tripeptide was synthesized separately in solution.

Following the previously reported strategy (Zabrocki et al., 1988; 1992; Nachman et al., $2002 \mathrm{~b})$, the fully protected dipeptide Z-D-Phe-D-Ala-OBzl was converted into the tetrazole analogue, Z-D-Phe- $\psi\left[\mathrm{CN}_{4}\right]$-D-AlaOBzl using $\mathrm{PCl}_{5}$ and hydrazoic acid in the presence of quinoline with full retention of configuration at both chiral centers. Careful treatment of the dipeptide tetrazole analogue with $30 \% \mathrm{HBr}$ in acetic acid for $20 \mathrm{~min}$ gave the hydrobromide salt of H-D-Phe- $\psi\left[\mathrm{CN}_{4}\right]-\mathrm{D}$ -
Ala-OBzl. The hydrobromide was extended, from its N-terminus, by coupling with Boc$\mathrm{Phe}-\mathrm{OH}$ to yield the protected tripeptide Boc-Phe-D-Phe- $\psi\left[\mathrm{CN}_{4}\right]$-D-Ala-OBzl. A mixed anhydride (MA) procedure with isobutyl chloroformate was used as the method of choice to avoid diketopiperazine formation. Removal of the benzyl ester group from the tripeptide by hydrogenolysis in methanol over $10 \% \mathrm{Pd}$ on charcoal led to free acid BocPhe-D-Phe- $\psi\left[\mathrm{CN}_{4}\right]-\mathrm{D}-\mathrm{Ala}-\mathrm{OH}$. This peptide was used in the solid phase peptide synthesis procedure. The known proclivity of the C-terminal $\alpha$-carbon of the tetrazole dipeptide to epimerize (Yu \& Johnson, 1987; Zabrocki et al., 1992) under prolonged exposure to base led us to omit the neutralization step in the solid phase synthesis of the peptide containing the tetrazole unit.

Following cleavage from the resin by means of trifluoromethanesulfonic acid, the peptidomimetic analogue was purified on a Delta-pak $\mathrm{C}_{18}\left(t_{\mathrm{R}}=19.5 \mathrm{~min}\right)$ and Watpro I125 column $\left(t_{\mathrm{R}}=5.8 \mathrm{~min}\right)$ under previously described conditions (Nachman et al., 2002b). MALDITOF-MS: $651.3\left(\mathrm{MH}^{+}\right)$. Amino acid analysis: F[1.0], G[0.8] (the tetrazole unit was not hydrolyzed under the analysis conditions).

NMR spectroscopy/molecular dynamics calculations. NMR spectra were acquired on a Bruker ARX500 $500 \mathrm{MHz}$ instrument, as previously described (Nachman et al., 2002b). In brief, samples were about 1 mmole in 10\% $\mathrm{D}_{2} \mathrm{O}$, water suppression was by presaturation, and a $5 \mathrm{~mm} \mathrm{HCN}$ probe was used. Coupling constants were measured from 1-D spectra. Most peak assignments were made using TOCSY spectra, but ambiguous peaks from amino acids containing aromatic moieties required intra-residue ROESY peaks for final determination.

Molecular modeling was performed using Sybyl Tripos software with modifications developed in our laboratories, running on SGI Indigo and $\mathrm{O} 2$ computers, as previously described (Nachman et al., 2002b; Moyna et al., 1999). In brief, distance constraints were de- 
termined from intensities of ROESY peaks measured by number of contours, with strong set to 1.8-2.7 $\AA$, medium 1.8-3.3 $\AA$ and weak 1.8-5 A. Simulated annealing consisted of a suitable number of heating cycles to 1000 deg. for $1000 \mathrm{fs}$, then exponential cooling to 200 deg over 1000 fs. After energy minimization, 5 or 10 lowest energy forms were superimposed and rms differences were calculated. Distance geometry allowed production of greatly differing starting conformers for the above calculations, preventing detection of only local minima. Tripos or Wiener et $a l$. force field was used for energy calculations.

Biology. The diuretic assay has been described in detail elsewhere (Coast, 1998). In brief, Malpighian tubules were removed from adult female crickets $6-12$ days old and were transferred to $5 \mu \mathrm{L}$ drops of bathing solution having the following composition (in mmoles/L): $\mathrm{NaCl}, 82 ; \mathrm{KCl}, 27 ; \mathrm{CaCl}_{2}, 2 ; \mathrm{Mg}$ $\mathrm{Cl}_{2}$, 8.5; $\mathrm{NaH}_{2} \mathrm{PO}_{4}, 4 ; \mathrm{NaOH}, 11$; glucose, 24; proline, 10; Hepes, 25. The $\mathrm{pH}$ was adjusted
40 min periods before and after the addition of the peptide analogue. Diuretic activity is calculated as the increase in fluid secretion ( $\Delta \mathrm{nl} / \mathrm{mm}$ per $\mathrm{min}$ ) and is expressed as a percentage of the response to a supramaximal dose $(10 \mathrm{nM})$ of achetakinin-I assayed on Malpighian tubules taken from the same insect.

\section{RESULTS AND DISCUSSION}

Previous evaluation of the insect kinin analogue Phe-Phe- $\psi\left[\mathrm{CN}_{4}\right]$-Ala-Trp-Gly- $\mathrm{NH}_{2}$, incorporating a tetrazole moiety $\left(\psi\left[\mathrm{CN}_{4}\right]\right.$; Fig. 1), in a cricket diuretic assay demonstrated significant activity retention in the physiological range, with an $\mathrm{EC}_{50}$ of $3.4 \times$ $10^{-7} \mathrm{M}$ and a $100 \%$ maximal response (Table 1). The (L-Phe ${ }^{2}, \mathrm{D}-\mathrm{Ala}^{3}$ )('LD') stereochemical variant demonstrated partial antagonism of natural insect kinins, limiting the diuretic response to $50 \%$ of maximal (Nachman et al., 2002b). The $\mathrm{EC}_{50}$ of the partial antagonist activity of the 'LD' stereochemical vari-

Table 1. Diuretic activity of peptidomimetic tetrazole insect kinin analogues in the cricket Acheta domesticus

\begin{tabular}{|c|c|}
\hline Tetrazole analog & $\begin{array}{l}\text { Stimulation of Malpighian tubule } \\
\text { fluid secretion }-\mathrm{EC}_{50}\left(10^{-7} \mathrm{M}\right) \\
\text { (\% Maximal response) }\end{array}$ \\
\hline Phe-L-Phe- $\psi\left[\mathrm{CN}_{4}\right]-\mathrm{L}-\mathrm{Ala}-\mathrm{Trp}-\mathrm{Gly}-\mathrm{NH}_{2} \quad$ ('LL’) & (Nachman et al., 2002b) \\
\hline Phe-L-Phe- $\psi\left[\mathrm{CN}_{4}\right]-\mathrm{D}-\mathrm{Ala}-\mathrm{Trp}-\mathrm{Gly}-\mathrm{NH}_{2}$ ('LD’) & (Nachman et al., 2002b) \\
\hline Phe-D-Phe- $\psi\left[\mathrm{CN}_{4}\right]$-D-Ala-Trp-Gly-NH ${ }_{2}$ ('DD’) & $5.8(100)$ \\
\hline
\end{tabular}

${ }^{*}$ Partial antagonist. Natural insect kinin diuretic response is limited to $50 \%$ of maximal.

to 7.2 with $1 \mathrm{M} \mathrm{NaOH}$. The dissected tubules and associated saline droplets are held under liquid paraffin. Urine escapes from a cut made close to the proximal end of the tubule and collects as a discrete droplet in the paraffin. Urine samples are collected at intervals and their volume is determined from measurements of droplet diameter under a microscope. After a $40 \mathrm{~min}$ equilibration period, the rate of secretion was measured over two ant was $4.3 \times 10^{-7} \mathrm{M}$ (Table 1$)$. This partial antagonist activity indicated that the ' $\mathrm{LD}$ ' tetrazole analogue could also bind with, but could not fully activate, the insect kinin receptor. As the tetrazole is a mimic of a cis-peptide bond type VI $\beta$-turn, it provided definitive evidence for the active conformation of the insect kinins in the cricket diuretic assay.

Distance and angle constraints obtained from two-dimensional NMR spectroscopy on 
Phe-Phe- $\psi\left[\mathrm{CN}_{4}\right]$-D-Ala-Trp-Gly- $\mathrm{NH}_{2}$ in aqueous solution were incorporated into molecular dynamics calculations to yield low energy conformations. Full details on the NMR spectroscopic data and molecular dynamics calculations of this analogue will appear in a subsequent publication. In Fig. 2, the 'LD'-tetrazole insect kinin analogue in an open 1-4 turn conformation is superimposed upon the previously determined low-energy conformation electrostatic interaction that occurs between the side chains of the $\mathrm{Phe}^{1}$ and $\mathrm{Trp}^{4}$ side chains in the 'LL' tetrazole analogue; which allows these two critical side chains to present an aromatic surface to the receptor (Nachman et al., 2002b). This aromatic surface has been previously proposed to be an important element in the insect kinin receptor interaction model (Nachman et al., 1990; Roberts et al., 1997; Moyna et al., 1999). The

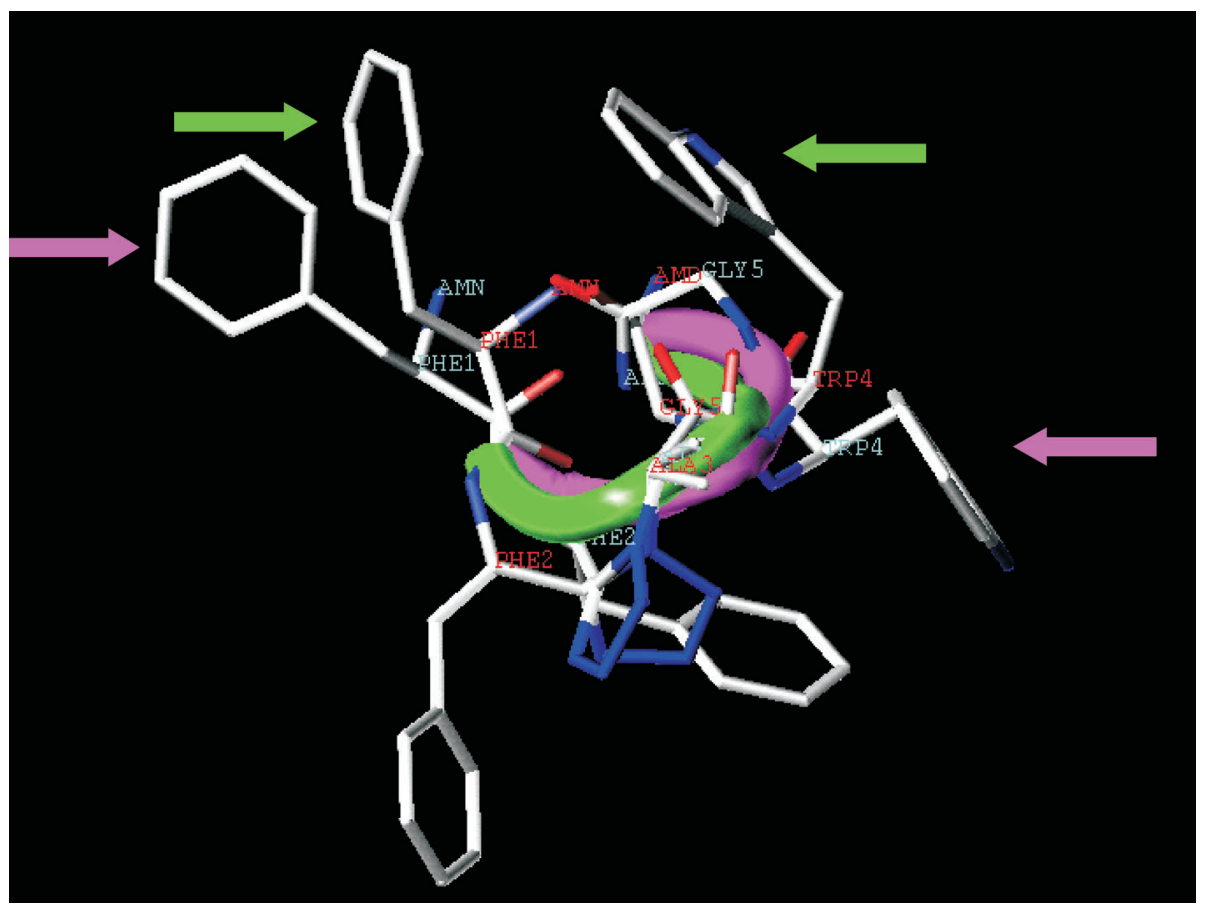

Figure 2. Superposition of the lowest energy conformations of 'LL' (green tube) and 'LD' (purple tube) stereochemical variants of the insect kinin tetrazole analogue Phe-Phe- $\psi\left[\mathrm{CN}_{4}\right]-\mathrm{Ala}-\mathrm{Trp}-\mathrm{Gly}_{\mathrm{N}} \mathrm{NH}_{2}$.

Both form an open turn over residues 1-4. The arrows (green/light gray = 'LL'; and purple/dark gray = 'LD') delineate the aromatic side chains of the critical residues $\mathrm{Phe}^{1}$ and $\operatorname{Trp}^{4}$ for each of the two stereochemical variants. The $\mathrm{Phe}^{1}{ }^{-} \operatorname{Trp}^{4}$ side chains of the 'LL' tetrazole analogue form an electrostatic interaction, leading to a common aromatic surface that is postulated to be an important component of the insect kinin-receptor interaction (Nachman et al., 1990). The $\mathrm{Phe}^{1}-\mathrm{Trp}^{4}$ side chains of the 'LD' tetrazole analogue do not form an electrostatic interaction as readily, and the analogue shows rather a preference for an electrostatic interaction between $\operatorname{Trp}^{4}$ and $\mathrm{Phe}^{2}$; the latter a non-critical residue.

for the 'LL'-tetrazole analogue (Nachman et al., 2002b). As can be seen in this figure, both analogs form a similar overall shape, an open turn over core residues 1-4; perhaps an indication as to why both are able to bind to the insect kinin diuretic receptor in the cricket. However, the change in stereochemistry of the $\alpha$-carbon at the $\mathrm{N}$-terminal end of the tetrazole moiety appears to interfere with the arrows in Fig. 2 designate the two sets of aromatic side chain moieties for each stereochemical variant. In the computer illustration (Fig. 2), the side chains of $\mathrm{Phe}^{1}$ and $\mathrm{Trp}^{4}$ in the ' $\mathrm{LD}$ ' analogue do not readily interact, whereas an electrostatic interaction between the $\operatorname{Trp}^{4}$ and $\mathrm{Phe}^{2}$ side chains is apparently preferred. This impaired ability to readily adopt an electrostatic interaction between 
$\mathrm{Phe}^{1}$ and $\operatorname{Trp}^{4}$ may explain why the 'LD' tetrazole analogue cannot fully activate the cricket diuretic receptor.

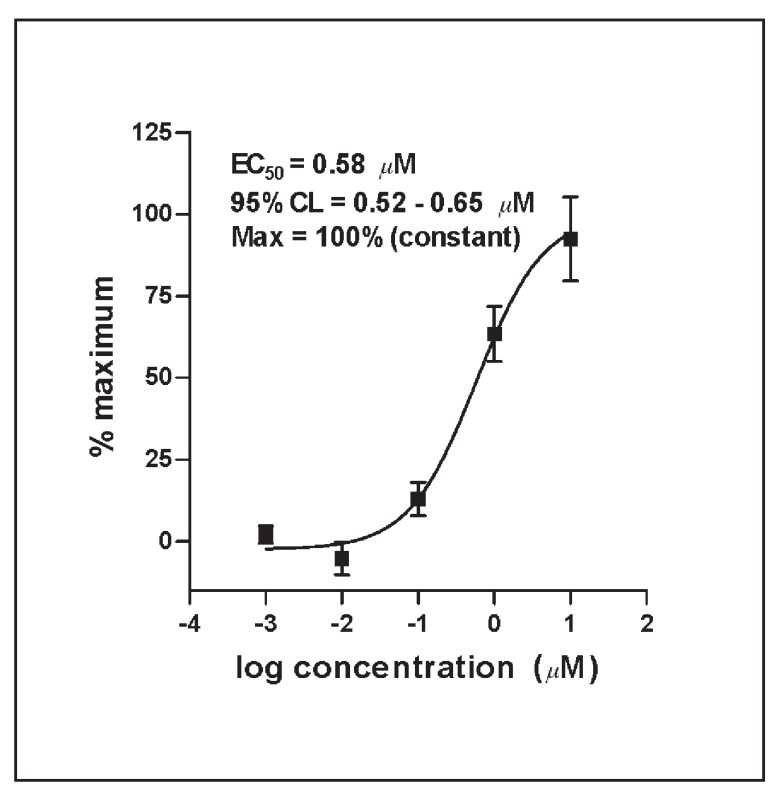

Figure 3. Dose-response curve for the insect kinin tetrazole analogue Phe-D-Phe- $\psi\left[\mathrm{CN}_{4}\right]-$ D-Ala-Trp- Gly- $\mathrm{NH}_{2}$ ('DD') in a cricket Malpighian tubule fluid secretion assay.

The activity is expressed as a percentage of the diuretic activity ( $\Delta \mathrm{nl} / \mathrm{min}$ ) of $10 \mathrm{nM}$ achetakinin-I. The sigmoidal dose-response curve with variable slope was fitted by non-linear regression using GraphPad Prism. Data points are the means of 6-8 determinations.

Following synthesis of the (D-Phe ${ }^{2}, \mathrm{D}-\mathrm{Ala}^{3}$ ) ('DD') version of the analogue PhePhe- $\psi\left[\mathrm{CN}_{4}\right]$-Ala-Trp-Gly- $\mathrm{NH}_{2}$, it was evaluated in the cricket diuretic assay and found to be an insect kinin agonist. It demonstrated stimulation of Malpighian tubule fluid secretion with an $\mathrm{EC}_{50}$ of $5.8 \times 10^{-7} \mathrm{M}$ (Table 1), a value which is slightly less than that of the 'LL' analogue, although the difference is not statistically significant. It is evident that 'DD' stereochemistry, unlike 'LD', is compatible with both binding and complete activation of the insect kinin receptor in the cricket bioassay. The aqueous solution structure of the 'DD' tetrazole analogue will be the subject of future investigation.
The results further delineate the structural and stereochemical requirements for both binding and activation of the insect kinin receptor on cricket Malpighian tubules, invaluable information for the development of biostable agonist and antagonist analogues of this important class of insect neuropeptides. Biostable peptidomimetic analogues can potentially disrupt the digestive and diuretic processes regulated by the insect kinins, and provide new candidates for future pest insect control agents.

\section{R E F E R E N C E S}

Coast GM. (1998) In Recent Advances in Arthropod Endocrinology. Coast GM, Webster SG, eds, pp 189-209. Cambridge University Press, Cambridge.

Holman GM, Nachman RJ, Coast GM. (1999) Peptides.; 20: 1-10.

Moyna G, Williams HJ, Nachman RJ, Scott AI. (1999) Biopolymers.; 49: 403-13.

Nachman RJ, Holman GM. (1991) In Insect Neuropeptides: Chemistry, Biology and Action. Menn JJ, Kelly TJ, Masler EP, eds, pp 194-214. American Chemical Society, Washington D.C.

Nachman RJ, Roberts VA, Holman GM, Tainer JA. (1990 In: Progress in Comparative Endocrinology. Epple A, Scanes CG, Stetson MH, eds, pp 60-6. Cambridge University Press, Cambridge.

Nachman RJ, Holman GM, Coast GM. (1998) In Recent Advances in Arthropod Endocrinology. Coast GM, Webster SG, eds, pp 379-391. Cambridge University Press, Cambridge.

Nachman RJ, Strey A, Isaac E, Pryor N, Lopez JD, Deng JG, Coast GM. (2002a) Peptides.; 23: $735-45$.

Nachman RJ, Zabrocki J, Olzcak J, Williams HJ, Moyna G, Scott AI, Coast GM. (2002b) Peptides.; 23: 709-16. 
Roberts VA, Nachman RJ, Coast GM, Hariharan M, Chung JS, Hoklman GM, Tainer J. (1997) Chem Biol.; 4: 105-17.

Yu KL, Johnson RL. (1987) J Org Chem.; 52: 2051-9.
Zabrocki J, Smith GD, Dunbar JB, Iijima H, Marshall GR. (1988) J Am Chem Soc.; 110: $5875-80$.

Zabrocki J, Dunbar JB, Marshall KW, Toth MV, Marshall GR. (1992) J Org Chem.; 57: 202-9. 